\title{
Realization of 70-nm T-gate InP-based PHEMT for MMW low noise applications
}

\author{
Wang Zhi-Ming ${ }^{1 \mathrm{a})}$, Lv Xin ${ }^{1}$, Hu Zhi-Fu ${ }^{2}$, Luo Xiao-Bin ${ }^{1}$, \\ Cui Yu-Xing ${ }^{2}$, Sun Xi-Guo ${ }^{2}$, Mo Jiang-Hui ${ }^{2}$, and Fu Xing-Chang ${ }^{2}$ \\ ${ }^{1}$ Beijing Key Laboratory of Millimeter Wave and Terahertz Technology, \\ Beijing Institute of Technology, Beijing 100081, China \\ ${ }^{2}$ National Key Laboratory of Application Specific Integrated Circuit (ASIC), \\ Hebei Semiconductor Research Institute, Shijiazhuang 050051, China \\ a)wangzhiming872@163.com
}

\begin{abstract}
T-gate InP-based low noise $\operatorname{In}_{0.52} \mathrm{Al}_{0.48} \mathrm{As} / \mathrm{In}_{0.65} \mathrm{Ga}_{0.35} \mathrm{As}$ pseudomorphic high electron mobility transistor (PHEMT) with well-balanced cutoff frequency $f_{t}$ and maximum oscillation frequency $f_{\max }$ were designed and fabricated. DC, RF, and noise characterizations are demonstrated. The maximum saturation current density $I_{d s s}$ and maximum extrinsic transconductance $g_{m, \max }$ are measured to be $894 \mathrm{~mA} / \mathrm{mm}$ and $1640 \mathrm{mS} / \mathrm{mm}$, respectively. And the extrapolated $f_{t}$ and $f_{\max }$ based on inflection point were $247 \mathrm{GHz}$ and $392 \mathrm{GHz}$, respectively. Due to the disadvantages of traditionally used $Y$-factor method, the new cold-source method was adopted to measure the noise parameters. The minimum noise figure $\left(N F_{\min }\right)$ is $1 \mathrm{~dB}$ at $30 \mathrm{GHz}$ associated with a gain of $15 \mathrm{~dB}$ at $V_{d s}$ of $0.8 \mathrm{~V}$ and $I_{d s}$ of $17 \mathrm{~mA}$. These excellent results make this InP-based PHEMT one of the most suitable devices for millimeter wave low noise applications.
\end{abstract}

Keywords: InP, PHEMTs, InAlAs/InGaAs, low noise, millimeter wave Classification: Microwave and millimeter wave devices, circuits, and systems

\section{References}

[1] P. Leuther, A. Tessmann, M. Dammann, C. Schworer, M. Schlechtweg, M. Mikulla, R. Losch and G. Weimann: 2007 International Conference on Indium Phosphide and Related Materials (2007) 24. DOI:10.1109/ICIPRM.2007. 380680

[2] A. Tessmann: IEEE J. Solid-State Circuits 40 (2005) 2070. DOI:10.1109/JSSC. 2005.854591

[3] D.-H. Kim and J. A. del Alamo: IEEE Electron Device Lett. 31 (2010) 806. DOI:10.1109/LED.2010.2051133

[4] D.-H. Kim and J. A. del Alamo: IEEE Electron Device Lett. 29 (2008) 830. DOI:10.1109/LED.2008.2000794

[5] W. Deal, X. B. Mei, K. M. K. H. Leong, V. Radisic, S. Sarkozy and R. Lai: IEEE Trans. THz Sci. Technol. 1 (2011) 25. DOI:10.1109/TTHZ.2011.2159539

[6] G. Meneghesso, A. Neviani, R. Oesterholt, M. Matloubian, T. Liu, J. J. Brown, C. Canali and E. Zanoni: IEEE Trans. Electron Dev. 46 (1999) 2. DOI:10.1109/ 
16.737434

[7] S. R. Bahl and J. A. del Alamo: IEEE Electron Device Lett. 13 (1992) 123. DOI: $10.1109 / 55.144979$

[8] R. Grundbacher, R. Lai, M. Barsky, R. Tsai, T. Gaier, S. Weinreb, D. Dawson, J. J. Bautista, J. F. Davis, N. Erickson, T. Block and A. Oki: 14th Indium Phosphide and Related Material conference, IPRM (2002) 455. DOI:10.1109/ ICIPRM.2002.1014466

[9] Y.-H. Zhong, X.-T. Wang, Y.-B. Su, Y.-X. Cao, Y.-M. Zhang, X.-Y. Liu and Z. Jin: Journal of Infrared and Millimeter Waves 32 [3] (2013) 193.

[10] Y.-H. Zhong, Y.-M. Zhang, Y.-M. Zhang, X.-T. Wang, H.-L. Lü, X.-Y. Liu and Z. Jin: Chin. Phys. B 22 (2013) 128503. DOI:10.1088/1674-1056/22/12/ 128503

[11] C. H. Liu, X. B. Mei, Y. C. Chou, L. S. Lee, J. M. Yang, M. Y. Nishimoto, P. H. Liu, R. To, A. Cavus, R. Tsai, M. Wojtowicz and R. Lai: 2009 Annual IEEE Compound Semiconductor Integrated Circuit Symposium (2009) 1. DOI:10. 1109/csics.2009.5315662

[12] L. Liu, A. R. Alt, H. Benedickter and C. R. Bolognesi: IEEE Electron Device Lett. 33 (2012) 209. DOI:10.1109/LED.2011.2176713

[13] Y. Zhong, X. Wang, Y. Su, Y. Cao, Z. Jin, Y. Zhang and X. Liu: J. Semicond. 33 (2012) 074004. DOI:10.1088/1674-4926/33/7/074004

[14] D. Smith, G. Dambrine and J.-C. Orlhac: Proc. of the 3rd European Microwave Integrated Circuits Conference (2008) 214. DOI:10.1109/EMICC.2008. 4772267

[15] Agilent Technologies: PNA-X Noise Figure FAQ [OL] (2014) http://www. home.agilent.com/agilent/editorial.jspx $? \mathrm{cc}=\mathrm{TW} \& \mathrm{lc}=\mathrm{cht} \& \mathrm{ckey}=2430705 \&$ nid $=-536902643.898624 \& \mathrm{id}=2430705$.

\section{Introduction}

In recent years, there has been an increasing demand for MMICs beyond $100 \mathrm{GHz}$ $[1,2]$. Due to the excellent high frequency and low noise performance, InP-based PHEMT is considered to be a unique candidate for applications beyond $100 \mathrm{GHz}$. Many high performance InP-based PHEMTs have been reported [1, 2, 3, 4, 5].

Excellent performance can be obtained by the combination of gate size scaling, parasitics reduction, and an increase of InAs mole fraction in the channel that improves electron transport properties. However, with In-content increased, the impact ionization effects will become serious due to the decreased energy band gap $E_{G}$, which have a number of negative consequences, such as reduced breakdown voltages, increase in output conductance and Kink effects, and permanent device degradation [6]. A method of increasing the effective energy band gap $E_{G, \text { eff }}$ in the channel is to introduce energy quantization by reducing the channel thickness to dimensions comparable to the electron wavelength $[6,7]$.

In this paper, InAs mole fraction has been increased to 0.65, the thickness of channel was reduced to $11-\mathrm{nm}$ for channel quantization, and the gate-length was reduced to $70 \mathrm{~nm}$. The design, fabrication and characteristics were described.

\section{Device structure and fabrication}

The epitaxial wafers were grown by molecular beam epitaxy (MBE) on 3-inch semi-insulating InP (100) substrates. The layer structure is shown in Fig. 1 and 
Table I. A room temperature electron mobility of $11,000 \mathrm{~cm}^{2} / \mathrm{v} \cdot \mathrm{s}$ has been achieved with a sheet charge of $3 \mathrm{E} 12 \mathrm{~cm}^{-2}$. As shown in Fig. 1, the gate electrode is located at an offset position from the center toward the source. The structural improvement of the offset gate due to a reduction in the distance between the source and gate enhances the cut-off frequency $\left(f_{t}\right)$ and the maximum stable gain $(M S G)$ by minimizing the gate-to-drain capacitance $\left(C_{g d}\right)$, as well as the source resistance $\left(R_{S}\right)$. In this devices, the space of source-drain and space of source-gate were $2 \mu \mathrm{m}$ and $0.8 \mu \mathrm{m}$, respectively.

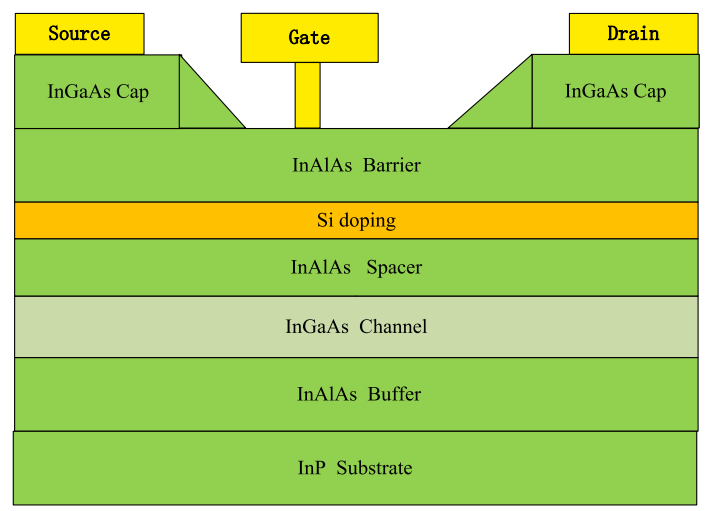

Fig. 1. InP-based PHEMT epitaxial structure

Table I. InP-based PHEMT epitaxial structure

\begin{tabular}{lccc}
\hline Layer & Material & Doping & Thickness \\
\hline Cap layer1 & $\mathrm{In}_{0.6} \mathrm{Ga}_{0.4} \mathrm{As}$ & $2 \times 10^{19} \mathrm{~cm}^{-3}$ & $10 \mathrm{~nm}$ \\
Cap layer2 & $\mathrm{In}_{0.53} \mathrm{Ga}_{0.47} \mathrm{As}$ & $5 \times 10^{18} \mathrm{~cm}^{-3}$ & $10 \mathrm{~nm}$ \\
Barrier layer & $\mathrm{In}_{0.52} \mathrm{Al}_{0.48} \mathrm{As}$ & undoped & $14 \mathrm{~nm}$ \\
Si $\delta$-doping layer & & $5 \times 10^{12} \mathrm{~cm}^{-2}$ & \\
Spacer layer & $\mathrm{In}_{0.52} \mathrm{Al}_{0.48} \mathrm{As}$ & undoped & $3 \mathrm{~nm}$ \\
Channel layer & $\mathrm{In}_{0.65} \mathrm{Ga}_{0.35} \mathrm{As}$ & undoped & $11 \mathrm{~nm}$ \\
Buffer layer & $\mathrm{In}_{0.52} \mathrm{Al}_{0.48} \mathrm{As}$ & undoped & $400 \mathrm{~nm}$ \\
& S.I.InP Substrate (100) & \\
\hline
\end{tabular}

The device fabrication were based on both optical and electron beam lithography (EBL). The transistor mesa was chemically wet etched to provide isolated active areas by removing an $\sim 200 \mathrm{~nm}$ thickness to expose the buffer layer, followed by the formation of ohmic contacts. The contact resistance $\left(R_{c}\right)$ of $0.035 \Omega \cdot \mathrm{mm}$ and the specific contact resistivity of $1.3 \times 10^{-7} \Omega \cdot \mathrm{cm}^{-2}$ were obtained by using Transmission Line Method (TLM). The gate recess was etched using a succinic acid based solution. The active devices feature T-shaped Pt-Ti-Pt-Au gates, which were defined by electron beam lithography in a three-layer resist (PMMA) process. Finally, the devices were passivated with 200-nm plasma enhanced chemical vapor deposited (PECVD) $\mathrm{Si}_{3} \mathrm{~N}_{4}$ for good reliability, robustness, low leakage current and high breakdown Voltage. 


\section{Device performances and discussions}

\subsection{DC characteristics}

The DC characteristics were measured by HP4142B semiconductor parameter analyzer. Fig. 2 shows the characteristics of drain current $\left(I_{d s}\right)$ versus drain-source voltage $\left(V_{d s}\right)$ of this device at room temperature.

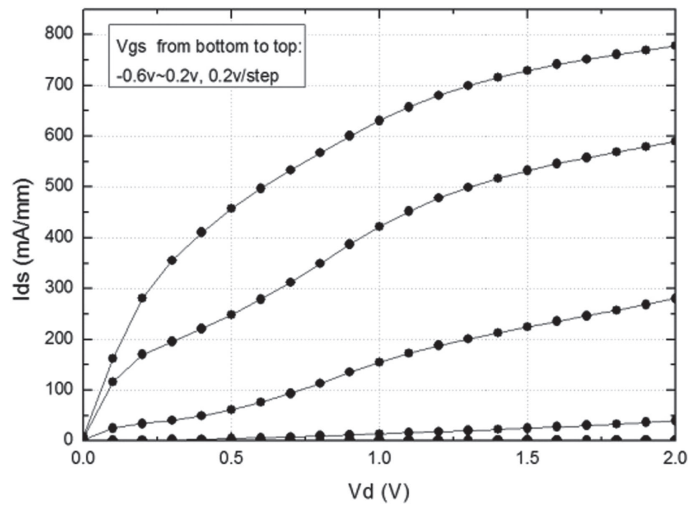

Fig. 2. DC characteristics of the device

Fig. 3 shows the characteristics of the $g_{m, \max }$ and the drain current $\left(I_{d s}\right)$ versus $V_{g s}$ at $V_{d s}$ of $1.5 \mathrm{~V}$. The maximum $g_{m}$ of the device at a $V_{d s}$ of $1.5 \mathrm{~V}$ and a $V_{g s}$ of $-0.1 \mathrm{~V}$ was $1640 \mathrm{mS} / \mathrm{mm}$. This high $g_{m}$ is due to the superior electron transport properties in the $\operatorname{In}_{0.65} \mathrm{Ga}_{0.35} \mathrm{As}$ channel and low ohmic contact resistance. A high drain current density of $894 \mathrm{~mA} / \mathrm{mm}$ was observed at a $V_{g s}$ of $0.6 \mathrm{~V}$.

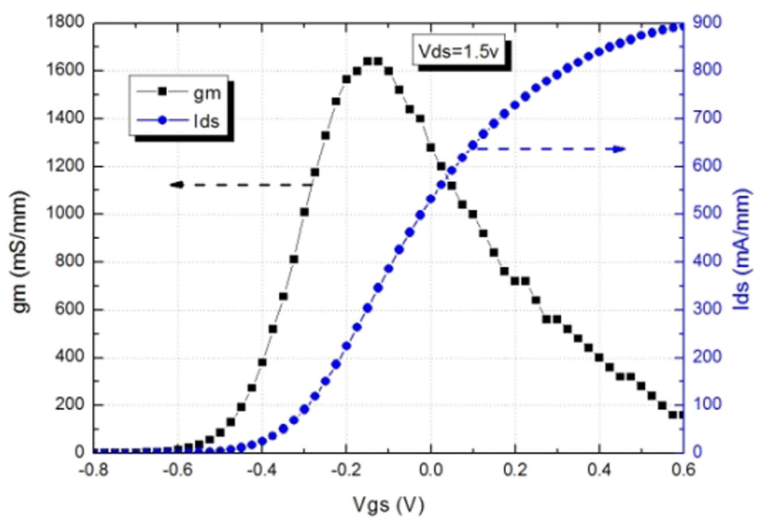

Fig. 3. $I_{d s}$ and $g_{m}$ versus $V_{g s}$

The off-state breakdown voltage ( $\left.B V_{\text {off-state }}\right)$ defined at a gate current of $1 \mathrm{~mA} /$ $\mathrm{mm}$ is $3.3 \mathrm{~V}$, which benefited from the $200-\mathrm{nm}$ thick $\mathrm{Si}_{3} \mathrm{~N}_{4}$ passivation layer and quantized channel. So the gate leakage current was very small, which was crucial for the lower frequency LNA applications since gate current was a contributing component to shot noise [8].

\subsection{RF characteristics}

The on-wafer RF measurements were performed by using Anritsu MS4647A series vector network analyzer and Anritsu 3743A frequency extender module 
$(70 \mathrm{KHz} 110 \mathrm{GHz})$ in National Institute of Metrology, China from 1 to $110 \mathrm{GHz}$ with $0.5 \mathrm{GHz} / \mathrm{step}$. And LRM (Line-Reflect-Match) method was used to calibrate the system full band from 1 to $110 \mathrm{GHz}$ at a time for the first time. So the discontinuities were improved than calibrations in separate frequency bands.

The measured $h_{21}$ gain, $M A G / M S G$ versus the frequency were shown in Fig. 4. The current gain $\left(H_{21}\right)$ decreases roughly with $-20 \mathrm{~dB} /$ decade slope as the frequency increases, so the value of the $f_{t}=247 \mathrm{GHz}$ was obtained by extrapolating $H_{21}$ to $0 \mathrm{~dB}$ with the same slope. However, power gain $M S G$ and $M A G$ is related to the stability factor $\mathrm{k}$ of the device, when $\mathrm{k}<1$, the maximum gain is $M S G$ which decreased with the slope of $-10 \mathrm{~dB} / \mathrm{decade}$, and when $\mathrm{k}>1$, the maximum gain is $M S G$ decreased with the slope of $-20 \mathrm{~dB} /$ decade.

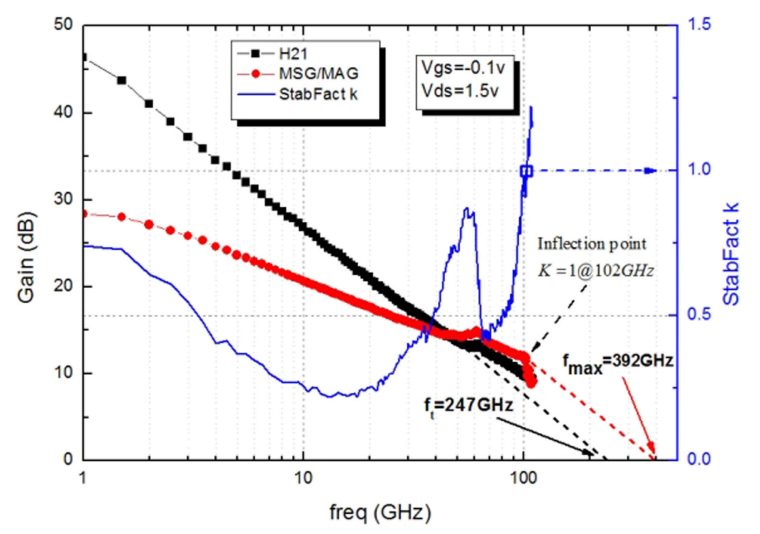

Fig. 4. Gain and the stability factor k versus frequency

As shown in Fig. 4, the inflection point $(\mathrm{k}=1)$ frequency was measured to be $102 \mathrm{GHz}$, where the $M A G$ of $\sim 11.7 \mathrm{~dB}$ was obtained, so the value of the $f_{\max }=$ $392 \mathrm{GHz}$ was obtained by extrapolating $M A G$ to $0 \mathrm{~dB}$ with $-20 \mathrm{~dB} /$ decade slop. Thus, the extrapolated $f_{\max }$ is much more accurate than those without measured inflection point $[2,9,10,13]$. Excellent DC and RF performances were shown in Table II.

Table II. Comparison with published HEMTs

\begin{tabular}{cccccc}
\hline Reference & $L_{g}(\mathrm{~nm})$ & $I_{d s s}(\mathrm{~mA} / \mathrm{mm})$ & $g_{m}(\mathrm{mS} / \mathrm{mm})$ & $f_{T} / f_{\max }(\mathrm{GHz})$ & Test range $(\mathrm{GHz})$ \\
\hline$[9]$ & 150 & 582 & 1052 & $151 / 303$ & $0 \sim 40$ \\
{$[10]$} & 150 & 681 & 952 & $164 / 390$ & $0 \sim 40$ \\
{$[11]$} & 100 & 850 & 1150 & $180 /-$ & - \\
{$[12]$} & 100 & 530 & 700 & $183 / 230$ & - \\
{$[2]$} & 100 & 900 & 1200 & $220 / 300$ & $1 \sim 110$ \\
{$[13]$} & 88 & 591 & 765 & $150 / 201$ & $0 \sim 40$ \\
{$[14]$} & 70 & 700 & 1600 & $300 / 300$ & - \\
{$[2]$} & 50 & 1300 & 1600 & $380 / 380$ & $1 \sim 110$ \\
This work & 70 & 894 & 1640 & $247 / 392$ & $1 \sim 110$ \\
\hline
\end{tabular}

\subsection{Noise characteristics}

An on-wafer noise parameters test is performed by using Agilent N5245A PNA-X series vector network analyzer and Maury Microwave MT984AU tunner. Due to frequency restriction of the tunner, the test frequency band is $8 \sim 45 \mathrm{GHz}$ with 
$0.2 \mathrm{GHz} / \mathrm{step}$. A new cold-source method was adopted to measure the noise figure of the device. This method eliminates two large sources of error: mismatch and noise-parameter effects [15], so this technique is much more accurate than the traditionally and widely used $Y$-factor method.

As is shown in Fig. 5, the $N F_{\text {min }}$ is less than $1.2 \mathrm{~dB}$ up to $40 \mathrm{GHz}$ at $V_{d s}$ of $0.8 \mathrm{~V}$ and $I_{d s}$ of $17 \mathrm{~mA}$. And as shown in Table III, an excellent $N F_{\min }$ of $1 \mathrm{~dB}$ is associated with a gain of $15 \mathrm{~dB}$ at $30 \mathrm{GHz}$.

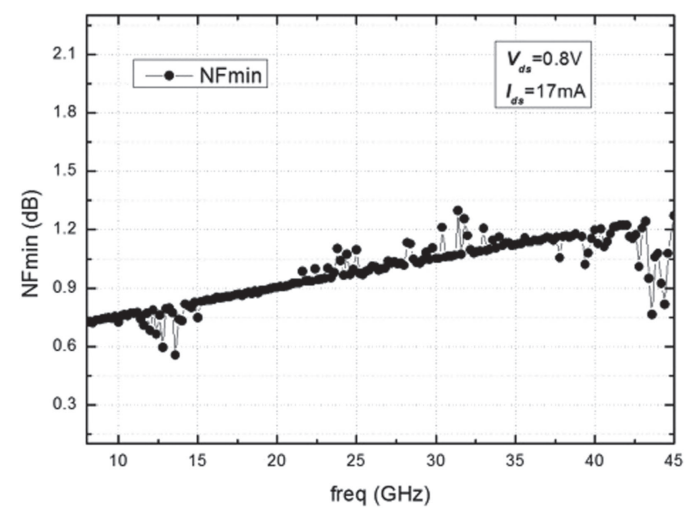

Fig. 5. $N F_{\min }$ of the device versus frequency

Table III. $N F_{\min }$ and $G_{a s s}$ Comparison with published HEMTs at $30 \mathrm{GHz}$

\begin{tabular}{ccccc}
\hline Reference & $L_{g}(\mathrm{~nm})$ & $f_{T} / f_{\max }(\mathrm{GHz})$ & $N F_{\min }(\mathrm{dB})$ & $G_{\text {ass }}(\mathrm{dB})$ \\
\hline$[14]$ & 130 & $105 / 105$ & 1.1 & 7.5 \\
{$[14]$} & 130 & $150 / 150$ & 0.83 & 11.7 \\
{$[14]$} & 70 & $300 / 300$ & 0.55 & 12.8 \\
This work & 70 & $247 / 392$ & 1 & 15 \\
\hline
\end{tabular}

From the above, in this work, DC and RF performances of this device were improved basically without sacrificing noise performance, so this InP-based PHEMT is one of the best devices for millimeter wave low noise applications.

\section{Conclusion}

70-nm InP-based PHEMTs have been designed and fabricated. Due to the scaling gate size and the high Indium composition in the channel, good performances have been achieved, including $I_{d s s}$ of $894 \mathrm{~mA} / \mathrm{mm}, g_{m, \max }$ of $1640 \mathrm{mS} / \mathrm{mm}, f_{t}$ and $f_{\max }$ of $247 \mathrm{GHz}$ and $392 \mathrm{GHz}$, respectively. The $N F_{\min }$ is $1 \mathrm{~dB}$ with associated gain of $15 \mathrm{~dB}$ at $30 \mathrm{GHz}$ and less than $1.2 \mathrm{~dB}$ up to $40 \mathrm{GHz}$. These excellent performances make this device suitable for millimeter-wave low noise applications.

\section{Acknowledgements}

This research is supported by the National Natural Science Foundation of China (Grant No. 61275107). The authors would like to thank Dr. Huang Hui from National Institute of Metrology, China for strong technical support on $1 \sim 110 \mathrm{GHz}$ full band test. And the authors also would like to thank the members of Hebei Semiconductor Research Institute for their help. 